\title{
Alopecia areata y Helicobacter pylori: una nueva asociación
}

\author{
Alopecia areata and Helicobacter pylori: a new association
}

Germán Campuzano-Maya, $M D^{1}$

$\mathbf{L}$

a alopecia areata es una enfermedad de los folículos pilosos con una fuerte evidencia

que apoya su origen autoinmune [1,2], aunque la patogénesis exacta no está aún lo suficientemente clara. La alopecia areata tiene una frecuencia que oscila entre el $0,7 \%$ y el $3,8 \%$ de los pacientes que acuden al dermatólogo, y del $2 \%$ en la población general; afecta a ambos sexos [3] y a menudo se informa una mayor concurrencia familiar [4, 5]. El patrón de pérdida del cabello puede variar y afectar cualquier parte del cuerpo.

En marzo de 2011 se presentó a la comunidad médica mundial el informe de un paciente con alopecia areata en quién las manifestaciones clínicas, en cuero cabelludo y barba, desaparecieron tras la erradicación exitosa de una infección por Helicobacter pylori, bajo el título "Cure of alopecia areata after eradication of Helicobacter pylori: A new association?» [6], en donde se comunicó una posible nueva asociación de esta enfermedad con Helicobacter pylori, hasta ese momento no descrita en la comunidad científica.

\section{Presentación del caso}

En el citado artículo se presentó el caso de un hombre de 43 años de edad con una historia de 8 meses de evolución, caracterizada por pérdida de pelo irregular en el cuero cabelludo y la barba (véase figura 1, secuencia A-B-C). El paciente consultó al dermatólogo quien le prescribió dexametasona $0,25 \%$ y minoxidil $5 \%$, de acuerdo con los estándares de manejo de la alopecia areata [7]. Además, recibió apoyo psiquiátrico con escitalopram $5 \mathrm{mg} /$ día, sin ninguna mejoría, pero sí con progresión de las lesiones.

Además de las manifestaciones dermatológicas antes citadas, debido a los antecedentes de dispepsia se le solicitó al paciente una prueba de aliento con urea marcada con carbono 13 (PAU-13 $\mathrm{CHp}^{\circledR}$ ), la cual arrojó un resultado, expresado en deltas de ${ }^{13} \mathrm{CO}^{2}\left(\delta^{13} \mathrm{CO}_{2}\right)$, positivo, con un valor de $6,95 \delta^{13} \mathrm{CO}_{2}$, considerándose que con esta prueba un resultado es negativo cuando está por debajo de $1,0 \delta^{13} \mathrm{CO}_{2}[8,9]$. Se hicieron estudios complementarios, inclui-

${ }^{1}$ Médico, especialista en Hematología y Patología Clínica. Docente Ad Honorem, Facultad de Medicina, Universidad de Antioquia. Coordinador Grupo de Investigación en Patología Clínica. Médico Director, Laboratorio Clínico Hematológico. Medellín, Colombia. Correo electrónico: gcm@lch.co Conflicto de intereses: el autor declara que no tiene conflicto de intereses Medicina \& Laboratorio 2017; 23: 149-158

Módulo 28 (Artículos de Reflexión), número 3. Editora Médica Colombiana S.A. $2017^{\circ}$ Recibido el 16 de marzo de 2017; aceptado el 17 de abril de 2017 
dos anticuerpos IgG contra Helicobacter pylori con un resultado de 52,4 unidades (valor de referencia < 9 unidades); además, anticuerpos antinucleares, anticuerpos contra las células parietales, anticuerpos contra el factor intrínseco, anticuerpos contra la peroxidasa tiroidea, TSH ultrasensible, T4 libre y T3 libre, con resultados en todos ellos negativos.

Se le indico un tratamiento de erradicación triconjugado de primera línea por 14 días, de acuerdo con las recomendaciones del Consenso de Maastricht III, con un inhibidor de la bomba de protones (omeprazol) $20 \mathrm{mg}$ dos veces al día, y dos antibióticos, amoxicilina $1 \mathrm{~g}$ dos veces al día y claritromicina $500 \mathrm{mg}$ dos veces al día [10]. Se le hizo seguimiento fotográfico de las lesiones y de la PAU $-{ }^{13} \mathrm{C} H p$ periódicamente de acuerdo con los protocolos de seguimiento de la respuesta a la erradicación [10]. La confirmación de la erradicación de Helicobacter pylori se obtuvo seis semanas después del tratamiento con un resultado negativo de la PAU${ }^{13} \mathrm{C} \mathrm{Hp}$ (valor $=0,81 \delta^{13} \mathrm{CO}_{2}$ ). A partir de la semana cuatro de haber terminado el tratamiento de erradicación se hizo evidente la reaparición del cabello en las zonas de alopecia areata en el cuero cabelludo y la desaparición de la lesión de la barba. En la secuencia A-B-C de la figura $\mathbf{1}$ se muestran las lesiones características de la alopecia areata en cuero cabelludo y barba; en la secuencia D-E-F de la citada figura, cuatro semanas poserradicación de Helicobacter pylori, se observa rebrote inicial de pelo en las lesiones iniciales y desaparición de la lesión en la barba; en la secuencia G-H-I de la misma figura, ocho semanas poserradicación de Helicobacter pylori, se observa que el pelo continúa creciendo en las áreas de cuero cabelludo afectado y que la barba permanece normal; en la secuencia J-K-L de la figura, 16 semanas poserradicación de Helicobacter pylori, se observa que las lesiones del cuero cabelludo han desaparecido y la barba se mantiene normal. Finalmente, en la secuencia $\mathbf{M}-\mathbf{N}-\mathbf{O}$ de dicha figura se observa el estado de las lesiones 44 semanas poserradicación de Helicobacter pylori [6].

\section{Nuevo informe de la asociación de la alopecia areata con Helicobacter pylori}

A principio de este año (2017), un estudio de casos y controles dio respuesta al interrogante ¿una nueva asociación? planteado en el artículo original [6], al confirmar estadísticamente la relación de la alopecia areata con la infección por Helicobacter pylori [11]. Este trabajo se realizó en la Facultad de Ciencias Médicas de la Universidad de Teherán (Teherán, Irán), e incluyó 81 pacientes con alopecia areata sin ningún tratamiento previo y 81 controles sanos. Mediante pruebas de aliento con urea marcada isotópicamente se estableció el estatus para Helicobacter pylori tanto en los pacientes con alopecia areata como en los controles sanos [11]. Se encontró que en la población de pacientes con alopecia areata 43 de 81 estaban infectados por Helicobacter pylori, lo cual representa una prevalencia de la infección en esta población de pacientes del 53,1\%, que contrasta con los 27 de los 81 controles sanos que se encontraban infectados por Helicobacter pylori, lo que indica una prevalencia de la infección por Helicobacter pylori en la población control del 33,3\%; estos datos son significativamente diferente, con un valor $p$ de 0,011 [11]. Desde el punto de los resultados estadísticamente significativos, esta sería la primera investigación en la que se demuestra la asociación de la infección por Helicobacter pylori con la alopecia areata, con un riesgo relativo de 2,263 (IC del 95\%: 1,199-4,273) [11]. 

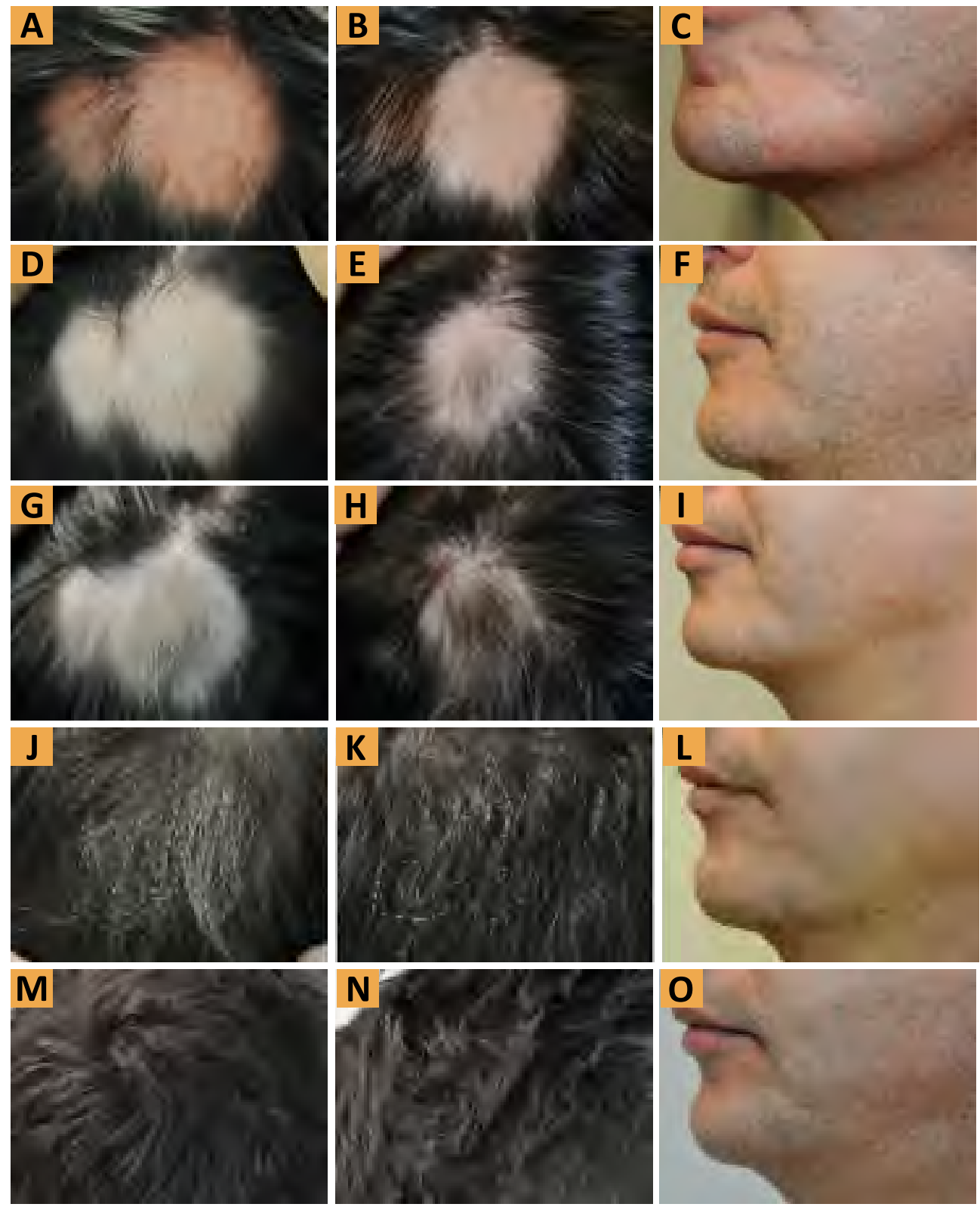

Figura 1. Secuencia fotográfica de lesiones antes y después de la erradicación de Helicobacter pylori. A-B-C: alopecia areata del cuero cabelludo (A y B) y barba (C) al momento del diagnóstico de la infección por Helicobacter pylori (semana 0), antes de su erradicación (PAU- ${ }^{13} \mathrm{C} \mathrm{Hp} \mathrm{6,95} \delta^{13} \mathrm{CO}_{2}$ ); D-E-F: evidencia de rebrote del cabello y desaparición de la lesión de la barba, cuatro semanas después de la erradicación; G-H-I: crecimiento del pelo en cuero cabelludo y barba libre de lesión a la semana 8 poserradicación (PAU_- ${ }^{13} \mathrm{C}$ Hp negativa $\left.0,81 \delta^{13} \mathrm{CO}_{2}\right)$; J-K-L: crecimiento del pelo en cuero cabelludo hasta desaparecer las lesiones y barba libre de lesión a la semana 16 poserradicación; $\mathbf{M}-\mathbf{N}-\mathbf{O}$ : crecimiento del pelo en cuero cabelludo hasta desaparecer las lesiones y barba libre de lesión a la semana 44 poserradicación (PAU- ${ }^{13} \mathrm{CHp}$ negativa $0,67 \delta^{13} \mathrm{CO}_{2}$ ). Tomado de "Cure of alopecia areata after eradication of Helicobacter pylori: a new association?" por G. Campuzano-Maya, 2011, World J Gastroenterol, 17 (26), p. 3166. CopyrightC 2011 por Baishideng [6]. 


\section{Discusión}

Hasta el momento se han descrito más de cincuenta manifestaciones extragástricas relacionadas, con mayor o menor grado de evidencia, con Helicobacter pylori [12-14] y, después de las manifestaciones hematológicas [15], hoy incluidas en las tres últimas versiones del Consenso de Maastricht [10, 16, 17] , las manifestaciones dermatológicas son unas de las asociaciones más frecuentes [18]. En el caso de la asociación de Helicobacter pylori con las enfermedades dermatológicas, entre las de mayor estudio e impacto clínico se incluyen la urticaria crónica [7, 19-25], la rosácea [26-35], la psoriasis [36-42], la púrpura de Schönlein-Henoch [43-49], la enfermedad de Behçet [50, 51], el prurigo nodularis [52], el prurigo cutáneo crónico [53], la esclerosis sistémica progresiva [54-57], el síndrome de Sjögren [58-62] y el síndrome de Sweet [63]; muchas de ellas mejorando o alcanzando remisión después de la erradicación de la infección por Helicobacter pylori $[18,20,37,42,52,64,65]$. A partir de los dos informes presentados [6, 11] se abre paso a la incorporación de la alopecia areata como una nueva manifestación extragástrica de la infección por Helicobacter pylori.

Al revisar la literatura médica mundial se evidencia que la alopecia areata y Helicobacter pylori presentan coexistencia con enfermedades de origen inmunológico comunes.

De un lado, la coexistencia de alopecia areata y tiroiditis autoinmunes [66-72]; de alopecia areata y vitíligo $[69,71,73-79]$, tiroiditis $[69,71,79]$ y dermatitis atópica [78, 80]; de alopecia areata y psoriasis [68, 73, 74, 81-99], enfermedad tiroidea [68] y policondritis [89]; de alopecia areata en pacientes con síndrome de Turner [96], síndrome de Down [99], síndrome de Sjögren [100] y trombocitopenia autoinmune, antes denominada púrpura trombocitopénica idiopática [101-103], entre otros.

Del otro, se ha documentado la asociación de Helicobacter pylori con tiroiditis autoinmunes [104-114], vitíligo [115] y anemia perniciosa [116-122], en algunos casos asociado también con enfermedad autoinmune de tiroides [117, 122], otras enfermedades autoinmunes como la artritis reumatoide [120], la infertilidad [118] y la deficiencia de vitamina $\mathrm{B}_{12}$, incluidas las modificaciones de la holotranscobalamina y la homocisteína $[123,124]$. También se ha documentado la coexistencia de Helicobacter pylori con el síndrome de Sjögren [58, 60-62], la psoriasis [36, 37, 42, 59, 125] y la trombocitopenia autoinmune [126-162], entre otras manifestaciones extragástricas de esta infección.

Estas coexistencias llevan a suponer que habría una relación, aún no establecida, entre la alopecia areata y la infección por Helicobacter pylori, la cual la comunidad científica tendrá como reto aclarar.

En relación con la etiología de la alopecia areata, aún no está plenamente establecida y sería un exabrupto aseverar que Helicobacter pylori sea la causa de esta: podría ser una nueva asociación o un factor que desencadene, a manera de "gatillo», las manifestaciones a nivel del folículo piloso. Uno de los mecanismos que con mayor posibilidad apuntan a explicar la asociación de la infección por Helicobacter pylori con la alopecia areata es el 
denominado mimetismo molecular. El mimetismo molecular se define como la similitud antigénica y funcional entre los componentes antigénicos microbianos y las moléculas del hospedero, que conduce a la producción de anticuerpos con reactividad cruzada [163]. En las infecciones por Helicobacter pylori se han descrito, o postulado, diversos autoantígenos con secuencias homólogas a elementos celulares o solubles de la bacteria que pueden ser blancos potenciales para el ataque por anticuerpos anti-Helicobacter pylori, como es el caso de la bomba $\mathrm{H}^{+} / \mathrm{K}^{+}$ATPasa de las células parietales que es atacada por anticuerpos anti-células parietales inducidos por componentes de la bacteria en la gastritis autoinmune y la anemia perniciosa [164-167], los antígenos de la superficie de las plaquetas, como la glicoproteína IIb/IIla (GP Ilb/IIla) y la glicoproteína I (GP I), que son reconocidos por anticuerpos dirigidos contra factores de virulencia de Helicobacter pylori como el VacA, el CagA y la ureasa B en la trombocitopenia inmune [126-131] y, posiblemente, componentes de los folículos pilosos con homología con antígenos de la bacteria que explicarían la alopecia areata en los pacientes infectados.

\section{Conclusión}

A modo de conclusión o reflexión final, de existir esta asociación, muy similar a como hoy se acepta con otras enfermedades dermatológicas, como la urticaria crónica, la rosácea, la psoriasis, la púrpura de Schönlein-Henoch, la enfermedad de Behçet, el prurigo nodularis, el prurigo cutáneo crónico, la esclerosis sistémica progresiva, el síndrome de Sjögren y el síndrome de Sweet, en donde que Helicobacter pylori, independiente del mecanismo o los mecanismos por los cuales induce el desarrollo, actúa como "gatillo», y su erradicación, cuando está presente, va seguida de mejora o resolución de la enfermedad de base, como ampliamente se ha referenciado en la literatura médica mundial.

Incorporar al diagnóstico y manejo de la alopecia areata la detección y erradicación de la infección por Helicobacter pylori, como se desprende de estos estudios iniciales, además de brindarle a algunos pacientes los beneficios de la resolución de la enfermedad asociada, muy similar a las situaciones previamente referenciadas en que se ha establecido la relación, desde el punto de vista clínico y de medicina preventiva, se estaría erradicando una bacteria altamente carcinogénica $[168,169]$, potencialmente prevenible con su erradicación [170, 171], sobre todo en los países como el nuestro en donde el cáncer gástrico lidera las estadísticas de mortalidad por cáncer en hombres y en mujeres con cerca de 5.000 muertes por año [172], y la fuente de la mayoría de las enfermedades ácido-pépticas, en particular de la úlcera péptica duodenal [173] y, en menor proporción, de la úlcera péptica gástrica [173], entre otras complicaciones plenamente demostradas en los portadores de esta infección, y desde el punto de vista de la salud pública se estaría reduciendo la prevalencia de Helicobacter pylori en el medio al eliminar la principal fuente de contaminación, la vía fecal, del medio ambiente y de nuevos pacientes a colonizar, de tal manera que lo que hoy es un círculo vicioso alrededor de la infección por Helicobacter pylori y sus complicaciones se convierta en un círculo virtuoso alejado de la infección y, por ende, de sus complicaciones. 


\section{Bibliografía}

1. Rosenstein ED, Warshauer BL. Alopecia areata and autoimmunity. J Am Acad Dermatol 2010;62:1065.

2. Islam N, Leung PS, Huntley AC, Gershwin ME. The autoimmune basis of alopecia areata: a comprehensive review. Autoimmun Rev 2015;14:81-89.

3. Tan E, Tay YK, Goh CL, Chin Giam Y. The pattern and profile of alopecia areata in Singapore--a study of 219 Asians. Int J Dermatol 2002;41:748-753.

4. Treem WR, Veligati LN, Rotter JI, Targan SR, Hyams JS. Ulcerative colitis and total alopecia in a mother and her son. Gastroenterology 1993;104:1187-1191.

5. Goh C, Finkel M, Christos PJ, Sinha AA. Profile of 513 patients with alopecia areata: associations of disease subtypes with atopy, autoimmune disease and positive family history. J Eur Acad Dermatol Venereol 2006;20:1055-1060.

6. Campuzano-Maya G. Cure of alopecia areata after eradication of Helicobacter pylori: a new association? World J Gastroenterol 2011;17:3165-3170.

7. MacDonald Hull SP, Wood ML, Hutchinson PE, Sladden M, Messenger AG, British Association of D. Guidelines for the management of alopecia areata. Br J Dermatol 2003;149:692-699.

8. Campuzano-Maya G. An optimized ${ }^{13} \mathrm{C}$-urea breath test for the diagnosis of $\mathrm{H}$ pylori infection. World J Gastroenterol 2007;13:5454-5464.

9. Campuzano-Maya G. Prueba de aliento optimizada con urea marcada con carbono 13 para la detección de Helicobacter pylori (PAU-13C Hp ${ }^{\circledR}$ ). Medicina \& Laboratorio 2012;18:137-160.

10. Malfertheiner P, Megraud F, O'Morain C, Bazzoli F, El-Omar E, Graham D, et al. Current concepts in the management of Helicobacter pylori infection: the Maastricht III Consensus Report. Gut 2007;56:772-781.

11. Behrangi E, Mansouri P, Agah S, Ebrahimi Daryani N, Mokhtare M, Azizi Z, et al. Association between Helicobacter Pylori infection and alopecia areata: A study in iranian population. Middle East Journal of Digestive Diseases 2017;9:107-110.

12. Mitchell HM, Kaakoush NO, Sutton P. Extragastric manifestations of Helicobacter pylori infection In: Helicobacter pylori in the twenty-first century, edited by Sutton P, Mitchell HM. UK: CPI Antony Rowe, Chipenham, 2010, p. 69-93.

13. Pacifico L, Osborn JF, Tromba V, Romaggioli S, Bascetta S, Chiesa C. Helicobacter pylori infection and extragastric disorders in children: a critical update. World J Gastroenterol 2014;20:1379-1401.

14. Kyburz A, Muller A. Helicobacter pylori and extragastric diseases. Curr Top Microbiol Immunol 2017;400:325-347.

15. Campuzano-Maya G. Hematologic manifestations of Helicobacter pylori infection. World J Gastroenterol 2014;20:12818-12838.

16. Malfertheiner P, Megraud F, O'Morain CA, Atherton J, Axon AT, Bazzoli F, et al. Management of Helicobacter pylori infection-the Maastricht IV/Florence Consensus Report. Gut 2012;61:646-664.

17. Malfertheiner P, Megraud F, O'Morain CA, Gisbert JP, Kuipers EJ, Axon AT, et al. Management of Helicobacter pylori infectionthe Maastricht V/Florence Consensus Report. Gut 2017;66:6-30.

18. Hernando-Harder AC, Booken N, Goerdt S, Singer MV, Harder H. Helicobacter pylori infection and dermatologic diseases. Eur J Dermatol 2009.

19. Galadari IH, Sheriff MO. The role of Helicobacter pylori in urticaria and atopic dermatitis. Skinmed 2006;5:172-176.

20. Abdou AG, Elshayeb EI, Farag AG, Elnaidany NF. Helicobacter pylori infection in patients with chronic urticaria: correlation with pathologic findings in gastric biopsies. Int J Dermatol 2009;48:464-469.

21. Shakouri A, Compalati E, Lang DM, Khan DA. Effectiveness of Helicobacter pylori eradication in chronic urticaria: evidence-based analysis using the Grading of Recommendations Assessment, Development, and Evaluation system. Curr Opin Allergy Clin Immunol 2010;10:362-369.

22. Bruscky DM, da Rocha LA, Costa AJ. Recurrence of chronic urticaria caused by reinfection by Helicobacter pylori. Rev Paul Pediatr 2013;31:272-275.

23. Rasooly MM, Moye NA, Kirshenbaum AS. Helicobacter pylori: A significant and treatable cause of chronic urticaria and angioedema. Nurse Pract 2015;40:1-6.

24. Mogaddam MR, Yazdanbod A, Ardabili NS, Maleki N, Isazadeh S. Relationship between Helicobacter pylori and idiopathic chronic urticaria: effectiveness of Helicobacter pylori eradication. Postepy Dermatol Alergol 2015;32:15-20.

25. Gu H, Li L, Gu M, Zhang G. Association between Helicobacter pylori infection and chronic urticaria: A meta-analysis. Gastroenterol Res Pract 2015;2015:486974.

26. Rebora A, Drago F, Picciotto A. Helicobacter pylori in patients with rosacea. Am J Gastroenterol 1994;89:1603-1604.

27. Mini R, Figura N, D’Ambrosio C, Braconi D, Bernardini G, Di Simplicio F, et al. Helicobacter pylori immunoproteomes in case reports of rosacea and chronic urticaria. Proteomics 2005;5:777-787.

28. Szlachcic A, Sliwowski Z, Karczewska E, Bielanski W, Pytko-Polonczyk J, Konturek SJ. Helicobacter pylori and its eradication in rosacea. J Physiol Pharmacol 1999;50:777-786.

29. Mayr-Kanhäuser S, Kranke B, Kaddu S, Mullegger RR. Resolution of granulomatous rosacea after eradication of Helicobacter pylori with clarithromycin, metronidazole and pantoprazole. Eur J Gastroenterol Hepatol 2001;13:1379-1383.

30. Szlachcic A. The link between Helicobacter pylori infection and rosacea. J Eur Acad Dermatol Venereol 2002;16:328-333.

31. Díaz C, O'Callaghan CJ, Khan A, Ilchyshyn A. Rosacea: a cutaneous marker of Helicobacter pylori infection? Results of a pilot study. Acta Derm Venereol 2003;83:282-286.

32. Argenziano G, Donnarumma G, lovene MR, Arnese P, Baldassarre MA, Baroni A. Incidence of anti-Helicobacter pylori and anti-CagA antibodies in rosacea patients. Int J Dermatol 2003;42:601-604.

33. Zandi S, Shamsadini S, Zahedi MJ, Hyatbaksh M. Helicobacter pylori and rosacea. East Mediterr Health J 2003;9:167-171.

34. Boixeda de Miquel D, Vazquez Romero M, Vazquez Sequeiros E, Foruny Olcina JR, Boixeda de Miquel P, Lopez San Roman A, et al. Effect of Helicobacter pylori eradication therapy in rosacea patients. Rev Esp Enferm Dig 2006;98:501-509.

35. Dakovic Z, Vesic S, Vukovic J, Milenkovic S, Jankovic-Terzic K, Dukic S, et al. Ocular rosacea and treatment of symptomatic Helicobacter pylori infection: a case series. Acta Dermatovenerol Alp Panonica Adriat 2007;16:83-86. 
36. Ali M, Whitehead M. Clearance of chronic psoriasis after eradication therapy for Helicobacter pylori infection. J Eur Acad Dermatol Venereol 2008;22:753-754.

37. Martin Hübner A, Tenbaum SP. Complete remission of palmoplantar psoriasis through Helicobacter pylori eradication: a case report. Clin Exp Dermatol 2008;33:339-340.

38. Bhattarai S, Agrawal A, Rijal A, Majhi S, Pradhan B, Dhakal SS. The study of prevalence of Helicobacter pylori in patients with acne rosacea. Kathmandu Univ Med J (KUMJ) 2012;10:49-52.

39. El-Khalawany M, Mahmoud A, Mosbeh AS, F ABDA, Ghonaim N, Abou-Bakr A. Role of Helicobacter pylori in common rosacea subtypes: a genotypic comparative study of Egyptian patients. J Dermatol 2012;39:989-995.

40. Gravina A, Federico A, Ruocco E, Lo Schiavo A, Masarone M, Tuccillo C, et al. Helicobacter pylori infection but not small intestinal bacterial overgrowth may play a pathogenic role in rosacea. United European Gastroenterol J 2015;3:17-24.

41. Jorgensen AR, Egeberg A, Gideonsson R, Weinstock LB, Thyssen EP, Thyssen JP. Rosacea is associated with Helicobacter pylori: a systematic review and meta-analysis. J Eur Acad Dermatol Venereol 2017.

42. Campuzano-Maya G, Ruiz-Restrepo MC. Psoriasis of Helicobacter pylori infection. en proceso 2017.

43. Reinauer S, Megahed M, Goerz G, Ruzicka T, Borchard F, Susanto F, et al. Schönlein-Henoch purpura associated with gastric Helicobacter pylori infection. J Am Acad Dermatol 1995;33:876-879.

44. Machet L, Vaillant L, Machet MC, Buchler M, Lorette G. Schönlein-Henoch purpura associated with gastric Helicobacter pylori infection. Dermatology 1997;194:86.

45. Mozrzymas R, d’Amore ES, Montini G, Guariso G. Schönlein-Henoch vasculitis and chronic Helicobacter pylori associated gastritis and duodenal ulcer: a case report. Pediatr Med Chir 1997;19:467-468.

46. Cecchi R, Torelli E. Schönlein-Henoch purpura in association with duodenal ulcer and gastric Helicobacter pylori infection. J Dermatol 1998;25:482-484.

47. Fu KI, Yagi S, Mashimo Y, Sugitani K, Imamaki K, Yanagisawa M, et al. Regression of Helicobacter pylori-negative duodenal ulcers complicated by Schönlein-Henoch purpura with H. pylori eradication therapy: the first report. Dig Dis Sci 2005;50:381-384.

48. Grivceva-Panovska V, Grivceva Stardelova K, Serafimoski V. Henoch-Schönlein purpura in an adult patient: extragastric, cutaneous manifestation of Helicobacter pylori infection. Prilozi 2008;29:291-301.

49. Hoshino C. Adult onset Schönlein-Henoch purpura associated with Helicobacter pylori infection. Intern Med 2009;48:847-851.

50. Avci O, Ellidokuz E, Simsek I, Buyukgebiz B, Gunes AT. Helicobacter pylori and Behcet's disease. Dermatology 1999;199:140-143.

51. Imamura Y, Kurokawa MS, Yoshikawa H, Nara K, Takada E, Masuda C, et al. Involvement of Th1 cells and heat shock protein 60 in the pathogenesis of intestinal Behcet's disease. Clin Exp Immunol 2005;139:371-378.

52. Neri S, lerna D, D’Amico RA, Giarratano G, Leotta C. Helicobacter pylori and prurigo nodularis. Hepatogastroenterology 1999;46:2269-2272.

53. Kandyil R, Satya NS, Swerlick RA. Chronic pruritus associated with Helicobacter pylori. J Cutan Med Surg 2002;6:103-108.

54. Reinauer S, Goerz G, Ruzicka T, Susanto F, Humfeld S, Reinauer H. Helicobacter pylori in patients with systemic sclerosis: detection with the ${ }^{13} \mathrm{C}$-urea breath test and eradication. Acta Derm Venereol 1994;74:361-363.

55. Yazawa N, Fujimoto M, Kikuchi K, Kubo M, Ihn H, Sato S, et al. High seroprevalence of Helicobacter pylori infection in patients with systemic sclerosis: association with esophageal involvement. J Rheumatol 1998;25:650-653.

56. Danese S, Zoli A, Cremonini F, Gasbarrini A. High prevalence of Helicobacter pylori type I virulent strains in patients with systemic sclerosis. J Rheumatol 2000;27:1568-1569.

57. Farina G, Rosato E, Francia C, Proietti M, Donato G, Ammendolea C, et al. High incidence of Helicobacter pylori infection in patients with systemic sclerosis: association with Sicca Syndrome. Int J Immunopathol Pharmacol 2001;14:81-85.

58. Aragona P, Magazzu G, Macchia G, Bartolone S, Di Pasquale G, Vitali C, et al. Presence of antibodies against Helicobacter pylori and its heat-shock protein 60 in the serum of patients with Sjögren's syndrome. J Rheumatol 1999;26:1306-1311.

59. Sorrentino D, Faller G, DeVita S, Avellini C, Labombarda A, Ferraccioli G, et al. Helicobacter pylori associated antigastric autoantibodies: role in Sjögren's syndrome gastritis. Helicobacter 2004;9:46-53.

60. De Vita S, Ferraccioli G, Avellini C, Sorrentino D, Dolcetti R, Di Loreto C, et al. Widespread clonal B-cell disorder in Sjögren's syndrome predisposing to Helicobacter pylori-related gastric lymphoma. Gastroenterology 1996;110:1969-1974.

61. Nishimura M, Miyajima S, Okada N. Salivary gland MALT Iymphoma associated with Helicobacter pylori infection in a patient with Sjögren's Syndrome. J Dermatol 2000;27:450-452.

62. Theander E, Nilsson I, Manthorpe R, Jacobsson LT, Wadstrom T. Seroprevalence of Helicobacter pylori in primary Sjögren's syndrome. Clin Exp Rheumatol 2001;19:633-638.

63. Kürkçüoğlu N, Aksoy F. Sweet's syndrome associated with Helicobacter pylori infection. J Am Acad Dermatol 1997;37:123-124.

64. Di Campli C, Gasbarrini A, Nucera E, Franceschi F, Ojetti V, Sanz Torre E, et al. Beneficial effects of Helicobacter pylori eradication on idiopathic chronic urticaria. Dig Dis Sci 1998;43:1226-1229.

65. Wedi B, Kapp A. Helicobacter pylori infection in skin diseases: a critical appraisal. Am J Clin Dermatol 2002;3:273-282.

66. Cunliffe WJ, Hall R, Stevenson CJ, Weightman D. Alopecia areata, thyroid disease and autoimmunity. Br J Dermatol 1969;81:877881.

67. Seyrafi H, Akhiani M, Abbasi H, Mirpour S, Gholamrezanezhad A. Evaluation of the profile of alopecia areata and the prevalence of thyroid function test abnormalities and serum autoantibodies in Iranian patients. BMC Dermatol 2005;5:11.

68. Ishak RS, Piliang MP. Association between alopecia areata, psoriasis vulgaris, thyroid disease, and metabolic syndrome. J Investig Dermatol Symp Proc 2013;16:S56-57.

69. Diaz-Angulo S, Lopez-Hoyos M, Munoz-Cacho P, Lopez-Escobar M, Gonzalez-Lopez MA. High prevalence of thyroid autoimmunity in patients with alopecia areata and vitiligo: a controlled study. Australas J Dermatol 2015;56:142-143.

70. Noso S, Park C, Babaya N, Hiromine Y, Harada T, Ito H, et al. Organ specificity in autoimmune diseases: thyroid and islet autoimmunity in alopecia areata. J Clin Endocrinol Metab 2015;100:1976-1983.

71. Saylam Kurtipek G, Cihan FG, Erayman Demirbas S, Ataseven A. The frequency of autoimmune thyroid disease in alopecia areata and vitiligo patients. Biomed Res Int 2015;2015:435947.

72. Bin Saif GA. Severe subtype of alopecia areata is highly associated with thyroid autoimmunity. Saudi Med J 2016;37:656-661. 
73. Kumar P, Gupta A. Dermatoglyphic patterns in psoriasis, vitiligo and alopecia areata. Indian J Dermatol Venereol Lepro 2010;76:185-186.

74. Ghajarzadeh M, Ghiasi M, Kheirkhah S. Associations between skin diseases and quality of life: a comparison of psoriasis, vitiligo and alopecia areata. Acta Med Iran 2012;50:511-515.

75. Harris JE. Vitiligo and alopecia areata: apples and oranges? Exp Dermatol 2013;22:785-789.

76. Krishnaram AS, Saigal A, Adityan B. Alopecia areata - vitiligo overlap syndrome: an emerging clinical variant. Indian J Dermatol Venereol Leprol 2013;79:535-537.

77. Rork JF, Rashighi M, Harris JE. Understanding autoimmunity of vitiligo and alopecia areata. Curr Opin Pediatr 2016;28:463-469.

78. Drucker AM, Thompson JM, Li WQ, Cho E, Li T, Guttman-Yassky E, et al. Incident alopecia areata and vitiligo in adult women with atopic dermatitis: Nurses' Health Study 2. Allergy 2017;72:831-834.

79. Takahashi N, Sugaya M, Oka T, Miyagaki T, Sato S. Alopecia areata, thyroiditis and vitiligo vulgaris in a Japanese patient with smoldering type adult T-cell leukemia/lymphoma. J Dermatol 2017;44:e79-e80.

80. Mohan GC, Silverberg JI. Association of vitiligo and alopecia areata with atopic dermatitis: A systematic review and meta-analysis. JAMA Dermatol 2015;151:522-528.

81. Ronchese F. Psoriasis and alopecia areata in the same patient. R I Med J 1974;57:68-69.

82. Ganor S. Diseases sometimes associated with psoriasis. II. Alopecia areata. Dermatologica 1977;154:338-341.

83. Rosina P, Segalla G, Magnanini M, Chieregato C, Barba A. Turner's syndrome associated with psoriasis and alopecia areata. J Eur Acad Dermatol Venereol 2003;17:50-52.

84. Criado PR, Valente NY, Michalany NS, Martins JE, Romiti R, Aoki V, et al. An unusual association between scalp psoriasis and ophiasic alopecia areata: the Renbok phenomenon. Clin Exp Dermatol 2007;32:320-321.

85. Yang YC, Cheng YW, Lai CS, Chen W. Prevalence of childhood acne, ephelides, warts, atopic dermatitis, psoriasis, alopecia areata and keloid in Kaohsiung County, Taiwan: a community-based clinical survey. J Eur Acad Dermatol Venereol 2007;21:643-649.

86. Catanzaro D, Andrien M, Labbe M, Toungouz-Nevessignsky M. Computer-aided human leukocyte antigen association studies: a case study for psoriasis and severe alopecia areata. Hum Immunol 2010;71:783-788.

87. Ito T, Hashizume H, Takigawa M. Contact immunotherapy-induced Renbok phenomenon in a patient with alopecia areata and psoriasis vulgaris. Eur J Dermatol 2010;20:126-127.

88. Slowinska M, Kardynal A, Warszawik O, Czuwara J, Rudnicka L. Alopecia areata developing paralell to improvement of psoriasis during ustekinumab therapy. J Dermatol Case Rep 2010;4:15-17.

89. Murao K, Hida Y, Minato M, Kubo Y, Arase S. Relapsing polychondritis associated with psoriasis vulgaris and alopecia areata. Eur J Dermatol 2011;21:779-780.

90. Poot F, Antoine E, Gravellier M, Hirtt J, Alfani S, Forchetti G, et al. A case-control study on family dysfunction in patients with alopecia areata, psoriasis and atopic dermatitis. Acta Derm Venereol 2011;91:415-421.

91. Wylie GR, Burden D. Renbok phenomenon between psoriasis and alopecia areata. Clin Exp Dermatol 2011;36:816-817.

92. Khaled A, Hawilo A, Zaouak A, Zeglaoui F, Kharfi M, Kamoun MR. Association between scalp psoriasis and alopecia areata. Tunis Med 2012;90:344.

93. Navarro R, Dauden E, Gallo E, Santiago Sanchez-Mateos D, Garcia-Diez A. Alopecia areata during treatment of psoriasis with adalimumab and leflunomide: a case and review of the literature. Skin Pharmacol Physiol 2012;25:107-110.

94. Ono S, Miyachi Y, Arakawa A. Hair regrowth following TNF-alpha blockade in coexisting psoriasis vulgaris and alopecia areata. Eur J Dermatol 2013;23:537.

95. Ovcharenko Y, Serbina I, Zlotogorski A, Ramot Y. Renbok phenomenon in an alopecia areata patient with psoriasis. Int J Trichology 2013;5:194-195.

96. Dogruk Kacar S, Ozuguz P, Polat S. Coexistence of psoriasis, and alopecia areata with trachyonychia in a pediatric patient with Turner Syndrome. Arch Argent Pediatr 2014;112:e209-212.

97. Karia SB, De Sousa A, Shah N, Sonavane S, Bharati A. Psychiatric morbidity and quality of life in skin diseases: A comparison of alopecia areata and psoriasis. Ind Psychiatry J 2015;24:125-128.

98. Mrowietz U, Gerdes S, Glaser R, Schroder O. Successful Treatment of Refractory Alopecia Areata Universalis and Psoriatic Arthritis, But Not of Plaque Psoriasis with Tofacitinib in a Young Woman. Acta Derm Venereol 2017;97:283-284.

99. Schepis C, Siragusa M, Happle R. Psoriasis and alopecia areata in a Down syndrome patient: a Renbok phenomenon. Eur J Dermatol 2017;27:300-301.

100. Sato M, Saga K, Takahashi H. Postmenopausal frontal fibrosing alopecia in a Japanese woman with Sjögren's syndrome. J Dermatol 2008;35:729-731.

101. Levin RM, Travis SF, Heymann WR. Simultaneous onset of alopecia areata and idiopathic thrombocytopenic purpura: A potentia association? Pediatr Dermatol 1999;16:31-34.

102. Cohn SM, Buchanan GR. Immune thrombocytopenia and alopecia areata: spontaneous occurrence and resolution in the same patient. Pediatr Blood Cancer 2014;61:2110-2111.

103. Salman A, Eser A. Concurrence of immune thrombocytopenic purpura and alopecia areata: A rare association. Int J Trichology 2016;8:52-53.

104. De Luis DA, Varela C, de La Calle H, Canton R, de Argila CM, San Roman AL, et al. Helicobacter pylori infection is markedly increased in patients with autoimmune atrophic thyroiditis. J Clin Gastroenterol 1998;26:259-263.

105. Figura N, Di Cairano G, Loré F, Guarino E, Gragnoli A, Cataldo D, et al. The infection by Helicobacter pylori strains expressing CagA is highly prevalent in women with autoimmune thyroid disorders. J Physiol Pharmacol 1999;50:817-826.

106. Triantafillidis JK, Georgakopoulos D, Gikas A, Merikas E, Peros G, Sofroniadou K, et al. Relation between Helicobacter pylori infection, thyroid hormone levels and cardiovascular risk factors on blood donors. Hepatogastroenterology 2003;50 Suppl 2:cccxviii-cccxx.

107. Bertalot G, Montresor G, Tampieri M, Spasiano A, Pedroni M, Milanesi B, et al. Decrease in thyroid autoantibodies after eradication of Helicobacter pylori infection. Clin Endocrinol (Oxf) 2004;61:650-652.

108. Franceschi F, Satta MA, Mentella MC, Penland R, Candelli M, Grillo RL, et al. Helicobacter pylori infection in patients with Hashimoto's thyroiditis. Helicobacter 2004;9:369. 
109. Sterzl I, Hrda P, Potuznikova B, Matucha P, Hana V, Zamrazil V. Autoimmune thyroiditis and Helicobacter pylori--is there a connection? Neuro Endocrinol Lett 2006;27 Suppl 1:41-45.

110. Sterzl I, Hrda P, Matucha P, Cerovska J, Zamrazil V. Anti-Helicobacter pylori, anti-thyroid peroxidase, anti-thyroglobulin and anti-gastric parietal cells antibodies in Czech population. Physiol Res 2008;57 Suppl 1:S135-141.

111. Bassi V, Marino G, lengo A, Fattoruso O, Santinelli C. Autoimmune thyroid diseases and Helicobacter pylori: The correlation is present only in Graves's disease. World J Gastroenterol 2012;18:1093-1097.

112. Fiorini G, Zullo A, Castelli V, Lo Re G, Holton J, Vaira D. Role of Helicobacter pylori infection in the thyroid diseases. J Gastrointestin Liver Dis 2013;22:261-263.

113. Shi WJ, Liu W, Zhou XY, Ye F, Zhang GX. Associations of Helicobacter pylori infection and cytotoxin-associated gene A status with autoimmune thyroid diseases: A meta-analysis. Thyroid 2013;23:1294-1300.

114. Choi YM, Kim TY, Kim EY, Jang EK, Jeon MJ, Kim WG, et al. Association between thyroid autoimmunity and Helicobacter pylori infection. Korean J Intern Med 2017;32:309-313.

115. Dogan Z, Ozdemir P, Eksioglu M, Filik L. Relationship between Helicobacter pylori infection and vitiligo: A prospective study. Am J Clin Dermatol 2014

116. Howitz J, Schwartz M. Vitiligo, achlorhydria, and pernicious anaemia. Lancet 1971;1:1331-1334.

117. Fairfax AJ, Leatham A. Idiopathic heart block: association with vitiligo, thyroid disease, pernicious anaemia, and diabetes mellitus. Br Med J 1975;4:322-324.

118. Gulden KD. Pernicious anemia, vitiligo, and infertility. J Am Board Fam Pract 1990;3:217-220.

119. Held JL, Kohn SR. Vitiligo and pernicious anemia presenting as congestive heart failure. Cutis 1990;46:268-270.

120. Abraham Z, Rozenbaum M, Gluck Z, Feuerman EJ, Lahat N, Kinarty A. Vitiligo, rheumatoid arthritis and pernicious anemia. J Dermatol 1993;20:418-423.

121. Egli F, Walter R. Images in clinical medicine. Vitiligo and pernicious anemia. N Engl J Med 2004;350:2698.

122. Suzuki C, Hirai Y, Terui K, Kohsaka A, Akagi T, Suda T. Slowly progressive type 1 diabetes mellitus associated with vitiligo vulgaris, chronic thyroiditis, and pernicious anemia. Intern Med 2004;43:1183-1185.

123. Montes LF, Diaz ML, Lajous J, Garcia NJ. Folic acid and vitamin B12 in vitiligo: a nutritional approach. Cutis 1992;50:39-42.

124. Karadag AS, Tutal E, Ertugrul DT, Akin KO, Bilgili SG. Serum holotranscobalamine, vitamin B12, folic acid and homocysteine levels in patients with vitiligo. Clin Exp Dermatol 2012;37:62-64.

125. Mesquita PM, Diogo AF, Jorge MT, Berbert AL, Mantese SA, Rodrigues JJ. Relationship of Helicobacter pylori seroprevalence with the occurrence and severity of psoriasis. An Bras Dermatol 2017;92:52-57.

126. Takahashi T, Yujiri T, Shinohara K, Inoue Y, Sato Y, Fujii Y, et al. Molecular mimicry by Helicobacter pylori CagA protein may be involved in the pathogenesis of $\mathrm{H}$. pylori-associated chronic idiopathic thrombocytopenic purpura. Br J Haematol 2004;124:9196.

127. Kodama M, Kitadai $\mathbf{Y}$, Ito $\mathbf{M}$, Kai $\mathbf{H}$, Masuda H, Tanaka S, et al. Immune Response to CagA protein is associated with improved platelet count after Helicobacter pylori eradication in patients with idiopathic thrombocytopenic purpura. Helicobacter 2007;12:36-42.

128. Campuzano-Maya G. Proof of an association between Helicobacter pylori and idiopathic thrombocytopenic purpura in Latin America. Helicobacter 2007;12:265-273.

129. Stasi R, Provan D. Helicobacter pylori and Chronic ITP. Hematology Am Soc Hematol Educ Program 2008;2008:206-211.

130. Bai Y, Wang Z, Bai X, Yu Z, Cao L, Zhang W, et al. Cross-reaction of antibody against Helicobacter pylori urease B with platelet glycoprotein IIla and its significance in the pathogenesis of immune thrombocytopenic purpura. Int J Hematol 2009;89:142-149.

131. Banic M, Franceschi F, Babic Z, Gasbarrini A. Extragastric manifestations of Helicobacter pylori infection. Helicobacter $2012 ; 17$ Suppl 1:49-55.

132. Gasbarrini A, Franceschi F, Tartaglione R, Landolfi R, Pola P, Gasbarrini G. Regression of autoimmune thrombocytopenia after eradication of Helicobacter pylori. Lancet 1998;352:878.

133. Emilia G, Longo G, Luppi M, Gandini G, Morselli M, Ferrara L, et al. Helicobacter pylori eradication can induce platelet recovery in idiopathic thrombocytopenic purpura. Blood 2001;97:812-814.

134. Emilia G, Luppi M, Morselli M, Potenza L, D’Apollo N, Torelli G. Helicobacter pylori infection and idiopathic thrombocytopenic purpura. Br J Haematol 2002;118:1198-1199.

135. Kohda K, Kuga T, Kogawa K, Kanisawa Y, Koike K, Kuroiwa G, et al. Effect of Helicobacter pylori eradication on platelet recovery in Japanese patients with chronic idiopathic thrombocytopenic purpura and secondary autoimmune thrombocytopenic purpura. Br J Haematol 2002;118:584-588.

136. Veneri D, Franchini M, Gottardi M, D’Adda M, Ambrosetti A, Krampera M, et al. Efficacy of Helicobacter pylori eradication in raising platelet count in adult patients with idiopathic thrombocytopenic purpura. Haematologica 2002;87:1177-1179.

137. Hashino S, Mori A, Suzuki S, Izumiyama K, Kahata K, Yonezumi M, et al. Platelet recovery in patients with idiopathic thrombocytopenic purpura after eradication of Helicobacter pylori. Int J Hematol 2003;77:188-191.

138. Hino M, Yamane T, Park K, Takubo T, Ohta K, Kitagawa S, et al. Platelet recovery after eradication of Helicobacter pylori in patients with idiopathic thrombocytopenic purpura. Ann Hematol 2003;82:30-32.

139. Ando T, Tsuzuki T, Mizuno T, Minami M, Ina K, Kusugami K, et al. Characteristics of Helicobacter pylori-induced gastritis and the effect of $H$. pylori eradication in patients with chronic idiopathic thrombocytopenic purpura. Helicobacter 2004;9:443-452.

140. Nomura S, Inami N, Kanazawa S. The effects of Helicobacter pylori eradication on chemokine production in patients with immune thrombocytopenic purpura. Eur J Haematol 2004;72:304-305.

141. Sato K, Nagai T, Muroi K, Komatsu N, Ozawa K. [Helicobacter pylori eradication in patients with idiopathic thrombocytopenic purpura--the associateion between the activity of Helicobacter pylori and platelet recovery]. Rinsho Ketsueki 2004:45:12521254

142. Fujimura K. Helicobacter pylori infection and idiopathic thrombocytopenic purpura. Int J Hematol 2005;81:113-118.

143. Inaba T, Mizuno M, Take S, Suwaki K, Honda T, Kawai K, et al. Eradication of Helicobacter pylori increases platelet count in patients with idiopathic thrombocytopenic purpura in Japan. Eur J Clin Invest 2005;35:214-219.

144. Stasi R, Rossi Z, Stipa E, Amadori S, Newland AC, Provan D. Helicobacter pylori eradication in the management of patients with 
idiopathic thrombocytopenic purpura. Am J Med 2005;118:414-419.

145. Suzuki T, Matsushima M, Masui A, Watanabe K, Takagi A, Ogawa Y, et al. Effect of Helicobacter pylori eradication in patients with chronic idiopathic thrombocytopenic purpura-a randomized controlled trial. Am J Gastroenterol 2005;100:1265-1270.

146. Tsutsumi Y, Kanamori H, Yamato H, Ehira N, Kawamura T, Umehara S, et al. Randomized study of Helicobacter pylori eradication therapy and proton pump inhibitor monotherapy for idiopathic thrombocytopenic purpura. Ann Hematol 2005;84:807-811.

147. Veneri D, Krampera M, Franchini M. High prevalence of sustained remission of idiopathic thrombocytopenic purpura after Helicobacter pylori eradication: a long-term follow-up study. Platelets 2005;16:117-119.

148. Asahi A, Kuwana M, Suzuki H, Hibi T, Kawakami Y, Ikeda Y. Effects of a Helicobacter pylori eradication regimen on anti-platelet autoantibody response in infected and uninfected patients with idiopathic thrombocytopenic purpura. Haematologica 2006;91:1436-1437.

149. Sayan O, Akyol Erikci A, Ozturk A. The efficacy of Helicobacter pylori eradication in the treatment of idiopathic thrombocytopenic purpura--the first study in Turkey. Acta Haematol 2006;116:146-149.

150. Suvajdzic N, Stankovic B, Artiko V, Cvejic T, Bulat V, Bakrac M, et al. Helicobacter pylori eradication can induce platelet recovery in chronic idiopathic thrombocytopenic purpura. Platelets 2006;17:227-230.

151. Satake M, Nishikawa J, Fukagawa Y, Akashi K, Okamoto T, Yoshida T, et al. The long-term efficacy of Helicobacter pylori eradication therapy in patients with idiopathic thrombocytopenic purpura. J Gastroenterol Hepatol 2007;22:2233-2237.

152. Asahi A, Nishimoto T, Okazaki Y, Suzuki H, Masaoka T, Kawakami Y, et al. Helicobacter pylori eradication shifts monocyte Fcgamma receptor balance toward inhibitory FcgammaRIIB in immune thrombocytopenic purpura patients. J Clin Invest 2008;118:2939-2949.

153. Kong R, Qiu HC, Wu PF, Niu XH, Shen WX, Wang Y. [Clinical significance of Helicobacter pylori in pathogenesis of idiopathic thrombocytopenic purpura]. Zhongguo Shi Yan Xue Ye Xue Za Zhi 2008;16:1222-1226.

154. Rostami N, Keshtkar-Jahromi M, Rahnavardi M, Esfahani FS. Effect of eradication of Helicobacter pylori on platelet recovery in patients with chronic idiopathic thrombocytopenic purpura: A controlled trial. Am J Hematol 2008;83:376-381.

155. Rostami N, Keshtkar-Jahromi M, Rahnavardi M, Keshtkar-Jahromi M, Esfahani FS. Effect of eradication of Helicobacter pylori on platelet recovery in patients with chronic idiopathic thrombocytopenic purpura: a controlled trial. Am J Hematol 2008;83:376381.

156. Suzuki T, Matsushima M, Shirakura K, Koike J, Masui A, Takagi A, et al. Association of inflammatory cytokine gene polymorphisms with platelet recovery in idiopathic thrombocytopenic purpura patients after the eradication of Helicobacter pylori. Digestion 2008;77:73-78.

157. Scandellari R, Allemand E, Vettore S, Plebani M, Randi ML, Fabris F. Platelet response to Helicobacter pylori eradication therapy in adult chronic idiopathic thrombocytopenic purpura seems to be related to the presence of anticytotoxin-associated gene $\mathrm{A}$ antibodies. Blood Coagul Fibrinolysis 2009;20:108-113.

158. Tsumoto C, Tominaga K, Okazaki H, Tanigawa T, Yamagami H, Watanabe K, et al. Long-term efficacy of Helicobacter pylori eradication in patients with idiopathic thrombocytopenic purpura: 7-year follow-up prospective study. Ann Hematol 2009;88:789793.

159. Wu S, Li Y, Jian Z, Tang F. [Anti-Helicobacter pylori treatment in patients with idiopathic thrombocytopenic purpura]. Zhong Nan Da Xue Xue Bao Yi Xue Ban 2009;34:1251-1254.

160. Tag HS, Lee HS, Jung SH, Kim BK, Kim SB, Lee A, et al. Effects of Helicobacter pylori eradication in patients with immune thrombocytopenic purpura. Korean J Hematol 2010;45:127-132.

161. Sato R, Murakami K, Okimoto T, Watanabe K, Kodama M, Fujioka T. Development of corpus atrophic gastritis may be associated with Helicobacter pylori-related idiopathic thrombocytopenic purpura. Journal of gastroenterology 2011;46:991-997.

162. Payandeh M, Sohrabi N, Zare ME, Kansestani AN, Hashemian AH. Platelet count response to Helicobacter pylori eradication in iranian patients with idiopathic thrombocytopenic purpura. Mediterr J Hematol Infect Dis 2012;4:e2012056.

163. Chmiela M, Karwowska Z, Gonciarz W, Allushi B, Staczek P. Host pathogen interactions in Helicobacter pylori related gastric cancer. World J Gastroenterol 2017;23:1521-1540.

164. Amedei A, Bergman MP, Appelmelk BJ, Azzurri A, Benagiano M, Tamburini C, et al. Molecular mimicry between Helicobacter pylori antigens and $\mathrm{H}+, \mathrm{K}+$--adenosine triphosphatase in human gastric autoimmunity. J Exp Med 2003;198:1147-1156

165. Negrini R, Savio A, Appelmelk BJ. Autoantibodies to gastric mucosa in Helicobacter pylori infection. Helicobacter $1997 ; 2$ Supp 1:S13-16.

166. Toh BH, Chan J, Kyaw T, Alderuccio F. Cutting edge issues in autoimmune gastritis. Clinical reviews in allergy \& immunology 2010

167. Hershko C, Patz J, Ronson A. The anemia of achylia gastrica revisited. Blood Cells Mol Dis 2007;39:178-183.

168. Correa P. New strategies for the prevention of gastric cancer: Helicobacter pylori and genetic susceptibility. J Surg Onco 2005;90:134-138; discussion 138

169. Lee YC, Lin JT. Screening and treating Helicobacter pylori infection for gastric cancer prevention on the population level. J Gastroenterol Hepatol 2017;32:1160-1169.

170. Malfertheiner P, Sipponen P, Naumann M, Moayyedi P, Megraud F, Xiao SD, et al. Helicobacter pylori eradication has the potential to prevent gastric cancer: A State-of-the-Art Critique. Am J Gastroenterol 2005;100:2100-2115.

171. Fry LC, Monkemuller K, Malfertheiner P. Prevention of gastric cancer: a challenging but feasible task. Acta Gastroenterol Latinoam 2007;37:110-117.

172. Ferlay J, Soerjomataram I, Dikshit R, Eser S, Mathers C, Rebelo M, et al. Cancer incidence and mortality worldwide: sources, methods and major patterns in GLOBOCAN 2012. Int J Cancer 2015;136:E359-386.

173. Kuipers EJ, Thijs JC, Festen HP. The prevalence of Helicobacter pylori in peptic ulcer disease. Aliment Pharmacol Ther $1995 ; 9$ Suppl 2:59-69. 